\title{
A Taxonomy Of The Join Operations In The REA Data Model
}

Ting J. Wang (E-mail: tjwang@uwm.edu), University of Wisconsin-Milwaukee Ping Liu (E-mail: kate0707@ sina.com), East China Jiao Tong University

\begin{abstract}
The Resource-Event-Agent (REA) data model identifies these three categories of entities in business processes and establishes relationships among them based on the rules that underlay actual business practices. The model becomes more efficient when the principle of relational database design, i.e., normalization, is applied. However, the higher the level of normalization in the database, the higher will be the degree of information segregation. Therefore, to ensure the accuracy of the information retrieved, it is crucial to understand the database structure and apply queries with correct join operations. "Join" is one of the fundamental relational database query operations. Join handles the processes that determine how data from two tables will be merged and selected. In this paper, a taxonomy of the join operations applicable to the REA data model is presented: it classifies the combinations of the categorical components in the REA model, identifies the join operation, and links to AIS documents and reports.
\end{abstract}

\section{Introduction}

End-users are increasingly involved in querying databases to find and cope with the information needed to carry out their daily business activities (O'Donnell and David 2000). However, they are not equipped with the knowledge and skills that are essential for performing queries in relational database management systems (Bowen and Rohde 2002; Borthick et al. 2001; Chan et al. 1999). Database topics, such as database architecture, database management systems, and data modeling techniques, are covered to some extent in accounting education, but the textbooks written for the Accounting Information Systems (AIS) courses and covered by AIS educators often do not include sufficient coverage of information retrieval to provide the needed knowledge and skills (Bain, Blankley, and Smith 2002). Some studies provide basic information about database query, such as Structured Query Language (SQL) (Olsen 2000), and query guidelines (Pillsbury and Wang 2003). Nevertheless, a fundamental query language, "join", which specifies how data can be aggregated and selected, is never completely dealt with for AIS end-users.

The purpose of this paper is to provide AIS end-users and educators with knowledge about joins in relational database query, focusing on queries of information, such as accounting documents and financial reports in the $R E A$ model. We will provide a taxonomy of the join operations applicable to the $R E A$ data model to classify the combinations of the categorical components in the REA model, specify the type of join operation involved, and link to AIS documents and reports. We will demonstrate an REA data model by using a simple merchandise company case to illustrate applications of the join operations.

This paper is organized as follows: Section II reviews types of joins, Section III describes AIS applications of the join operations in the REA data model, and Section IV concludes the paper.

\section{Types Of Joins}

The REA data model has influenced industrial software and it plays a significant role in the AIS education (Weber 1986; Dunn and McCarthy 1997). The superiority of this data model over the traditional AIS architecture is documented in the literature for its ability to provide both financial and non-financial related information as well as improving data consistency and efficiency in storage (McCarthy 1982; Hollander, et al. 2000). 
REA data modeling in relational databases results in related information being decomposed and stored in separate tables. For example, information about business activities are taken apart and stored in different tables according to the Resources, Events, and Agents categories (e.g., Product, Selling Event, and Customer, respectively). Normalization of relations (entities/tables) will further decompose related information stored in these categorical tables. Subsequently, queries are used to merge tables and retrieve related information from the relational database. The join operation is required when there is more than one table involved in a query, combining tuples (records) from two different tables based on some common information.

The join operation has been examined and discussed extensively in the CIS and MIS literature. Most of these studies relate to algorithms of the join operations used to improve efficiency and optimization (Morishita 1997; Mishra and Eich 1992). There are more than a few join types, for example, equijoin, natural join, semijoin, outerjoin, and self-join (Mishra and Eich 1992), and most database textbooks in CIS and MIS include coverage of them (e.g., Ramakrishnan and Gehrke 2000).

To illustrate join operations, let's first take a look at the Cartesian product of two tables since it is related to some of these operations. The Cartesian product of two tables combines each record of the first table with every record of the second table. In other words, the derived (virtual/result) table of such Cartesian product consists of all combinations of records ( $\mathrm{N}$ x M) and attributes (data fields) from the two tables. For example, there are Customer and Zip Code tables as follows:

\begin{tabular}{|c|c|}
\hline \multicolumn{2}{|c|}{ CUSTOMER TABLE } \\
\hline Name & Zip Code \\
\hline Adam Smith & 53201 \\
\hline William King & 53211 \\
\hline
\end{tabular}

\begin{tabular}{|c|c|c|}
\hline \multicolumn{3}{|c|}{ ZIP CODE TABLE } \\
\hline ZipID & City & State \\
\hline 53201 & Milwaukee & WI \\
\hline 53706 & Madison & WI \\
\hline 54821 & Cable & WI \\
\hline
\end{tabular}

The result table of the Cartesian product is as follows:

\begin{tabular}{|c|c|c|c|c|}
\hline Name & Zip Code & ZipID & City & State \\
\hline Adam Smith & 53201 & 53201 & Milwaukee & WI \\
\hline Adam Smith & 53201 & 53706 & Madison & WI \\
\hline Adam Smith & 53201 & 54821 & Cable & WI \\
\hline William King & 53211 & 53201 & Milwaukee & WI \\
\hline William King & 53211 & 53706 & Madison & WI \\
\hline William King & 53211 & 54821 & Cable & WI \\
\hline
\end{tabular}

City and State give information about the customers, but they are stored in two different tables because they are violating the non-transitive dependency of the $3^{\text {rd }}$ norm form (i.e., the Zip Code determines the City and the State attributes). As a result, City, State, and ZipID are discomposed from the Customer table. The data values of the Zip Code and ZipID in Customer and Zip Code tables, respectively, are set to be inconsistent in order to ease the demonstration of different join operations (of course, the inconsistency could also be a result of lack of enforcing referential integrity ${ }^{1}$ between these two tables).

To perform a join operation, two data fields from the Cartesian product and a join condition must be specified in the query. Multiple conditions can be applied by using the logical connective AND (Desai 1990; El-Masri and Navathe 1989; Maier 1983). A general join is called a theta-join (or $\theta$-join). A $\theta$ operator, such as $=, \neq,>,<, \geq$, or $\leq$, will be required in every condition. A join operation is called an equijoin when a $\theta$ operator, "=", is specified in the condition. For example, a join involving the Customer and the Zip Code tables based on the equality between Zip Code and ZipID, is an equijoin. In our previous example, the result table of the equijoin will contain the first record of the Cartesian product since that is the only row in which the join condition is met (i.e., the data values of Zip Code and ZipID equal to each other). If a " $\neq$ " is used as a $\theta$ operator in a general join, the result table will include all but the first records in our example. 
The natural join can be defined as an equijoin, joining on same data fields in both tables and projecting on either one of data fields. In most cases, especially in the REA data model, one of the data fields is a key of one table and the other a foreign key created to link the tables, and the referential integrity is enforced on a 1-to-Many relationship. Our previous example of the Cartesian product does not fit in, as most cases do, because the referential integrity is not enforced (i.e., data values in Zip Code and ZipID are not consistent). Therefore, to demonstrate the natural join, suppose that the referential integrity were enforced (that is, a Zip Code value, 53211, is referenced in the Zip Code table with 53211, Shorewood, WI), the result table of the natural join will include both records from the Customer table with all the data fields from two tables except that one of the common data fields is (Zip Code or ZipID) dropped, as shown below.

\begin{tabular}{|c|c|c|c|}
\hline Name & Zip Code & City & State \\
\hline Adam Smith & 53201 & Milwaukee & WI \\
\hline William King & 53211 & Shorewood & WI \\
\hline
\end{tabular}

If the referential integrity is enforced between tables (which is not required in the natural join) with a 1-toMany relationship, the information obtained in the result table from the natural join will be based on the table that has the "Many" side of the relationship (i.e., the numbers of records are exactly the same). The natural join takes the table that has the "Many" side of the relationship and merges data from the table that has the "1" side of the relationship, based on the referenced values in the common data fields. In our modified example, there is a 1-to-Many relationship between the Zip Code and the Customer tables with the referential integrity enforced (i.e., all of the data values in the Zip Code, a foreign key in the Customer table, are referenced in the ZipID, a primary key in the Zip Code table). In the natural join, the result table will include all of the information from the Customer table (i.e., the basis of the information from the table with the "Many" side of the relationship) and then merge information about City and State from the Zip Code table based on the referenced values stored in the Zip Code and ZipID. In a case in which the referential integrity is not enforced, the basis of the information will still remain in the table that has the "Many" side of relationship, but the non-participative records will be excluded.

The semijoin operation is designed to exclude data fields of one table in the result table. It is similar to any join that projects only on data fields from one table. Semijoin reduces the size of the second table that is participating in the join operation and improves efficiency (Kambayashi 1985; Perrizo et al. 1989; Yoo and Lafortune 1989). For example, an equijoin joins on Zip Code and ZipID from Customer and Zip Code tables, respectively, and projects on Name and Zip Code data fields of the Customer table. It first drops the City and State data fields of the Zip Code table from the join operation and then selects records from the Cartesian product based on the equality (or any other $\theta$ operator) condition of Zip Code and ZipID. It will then exclude the joining data field, ZipID, before the result table is finalized.

The outerjoin operation (also called the external join) handles non-participative (or dangling) records from either or both tables. In any of the before-mentioned joins, records that are not included in the result table are called "non-participative records". For example, the second records in the Customer table, William King and 53211, and the second and the third records in the Zip Code table, 53706 and 54821, are non-participative records in the equijoin of the two tables on Zip Code and ZipID. There are three kinds of outerjoins: left-outerjoin, right-outerjoin, and full-outerjoin. In the result table, in addition to all participative records, the left-outerjoin includes the nonparticipative records from the left side of the table and the right-outerjoin includes the non-participative records from the right side of the table when the tables are placed next to one another in the query (e.g., SQL statement or Query-By-Example [QBE] screen). The full-outerjoin includes non-participative (in addition to participative) records from both tables. In our example, the left-outerjoin of Customer and Zip Code tables on Zip Code and ZipID for equality contains the first record from both tables and all non-participative records in the Customer table (as shown below), assuming the Customer table is on the left side and the Zip Code is on the right side. The nonparticipative records will contain null vales on the joining data fields from the table on the right.

\begin{tabular}{|l|l|l|l|c|}
\hline \multicolumn{1}{|c|}{ Name } & \multicolumn{1}{c|}{ Zip Code } & \multicolumn{1}{c|}{ ZipID } & \multicolumn{1}{c|}{ City } & State \\
\hline Adam Smith & 53201 & 53201 & Milwaukee & WI \\
\hline William King & 53211 & NULL & NULL & NULL \\
\hline
\end{tabular}


The self-join operation involves joining a table with the table itself. That is, a Cartesian product will be formed consisting of the combination of each record with all records of the same table. The selection of the records that takes place next is based on the data field(s) and the join condition using any of the $\theta$ operators. The self-join is performed when the relationship between records on a set of specific data fields in that table is of interest (e.g., we may be interested in the customer's purchasing behavior after they have purchased a specific product.)

Some of these join operations are required to retrieve disaggregated information from the database due to the $R-E-A$ categories and normalization, and some are used to manipulate the data for various purposes. However, the most commonly used join operations in the REA data model are natural and outer joins because the relationships between tables, if there are any, are pre-defined and pre-established in the data modeling. In the next section, we will use a simple merchandise company case to demonstrate the applications of these two join operations in the REA data model to retrieve accounting information, such as accounting documents and financial reports.

\section{AIS Applications Of Join Operations In The REA Model}

We created a simplified merchandise case similar to those in McCarthy (1979) and Wang, Du, and Lee (2002) in order to illustrate the taxonomy of the join operations in the REA data model. Table I shows the entities recognized within each type of business processes for a simplified merchandise company using the $R-E$ - $A$ categories in the REA data model. There is one Resources (Product), four consecutive Events (Purchase Ordering, Goods Receiving, Purchase Invoicing, and Purchase Payment Disbursing), and two Agents (Vendor and Employee) recognized in the Acquisition and Payment business processes, and one Resource (Product), four consecutive Events (Sales Ordering, Goods Shipping, Sales Invoicing, and Sales Collecting), and four Agents (Salesperson, Customer, Carrier, and Employee) in the Sales and Collection business processes. We eliminate the Cash Resource by assuming that there is only one cash account involved in the case. To simplify the case further, suppose that the purchaseinvoicing event is an optional entity in the database since most of its data can be obtained from the matched records between the goods receiving and purchase ordering entities.

Figure 1 depicts the REA data model, based on the entities recognized in Table I, and the cardinalities of relationships based on a generic merchandising case. We will also assume the types of relationships between entities in the data model, especially for those involving with Many-to-Many type (we will demonstrate the join operation dealing with a Many-to-Many relationship later in the paper). The layout of the REA data model in Figure 1 is organized using the types of business processes and the $R-E-A$ categories of the entities.

Table II shows the taxonomy of join operations in the REA data model by listing the entity categories involved, entity focused, join type based, origin of the information, and documents and reports generated in a query. There are many combinations of the three $R-E-A$ categories. However, in our example, there are two common types of combinations applicable to the accounting information systems, such as $R-E-A$ and Event-Event $(E-E)$. In the REA data model, most of the relationships are pre-defined and pre-established through the REA data modeling. As a result, the most commonly used join operations are Natural and Outer. Typical documents and reports often used by accounting users in their daily business activities, such as Purchase Order, Receiving Report, Purchase Journal, and Cash Disbursement Journal, are retrieved using these join operations.

The first major type of entity combination involves all three $R-E-A$ categories and has Event as the origin of the information that will require a Natural join in the query (see Table II). This type of join focuses on the Event and retrieves its related information from the Resources and Agents tables. The origin of Event-type information includes events such as Purchase Ordering, Goods Receiving, Purchase Disbursing, Sales Ordering, Goods Shipping, Sales Invoicing, and Sales Collecting. Documents are directly associated with Events since they are used to capture and/or process information involved in the Events. Reports are used to provide summarized information to users about the Events. Related events-documents-reports include Purchase Ordering-Purchase Order-Purchase Journal, Goods Receiving-Receiving Report-Receiving Summary, Purchase Disbursing-Check-Cash Disbursement Journal, etc.

For example, to obtain the Sales Journal, natural joins are performed involving Product (tblProduct), Sales Invoicing (tblSalesInvoicing), and Customer (tblCustomer) tables (see Figure 1 as well). A relationship table ${ }^{2}$ is 
created for the implementation of a Many-to-Many relationship between tblProduct and tblSalesInvoicing in relational database management systems. The join operation is straightforward in a 1-to-Many situation because it is not involved with an additional table. Figure 2 shows the process of the natural joins. In the joins, related information from tblProduct and tblCustomer ${ }^{3}$ are first aggregated with the information in the tblSalesInvoicing based on common information stored in the foreign keys ${ }^{4}$ (e.g., Customer\# in tblSalesInvoicing, and Product\# and Inv\# individually in the relationship table, tblSIProd) and then included in the result table. (See the note in Figure 2 for detailed descriptions of the join operations.) Figure 3 illustrates natural joins performed using both QBE and SQL. QBE has a graphical user interface that allows users to create queries by using example tables on the screen; it is often used as a more intuitive user-interface for simpler queries while SQL is utilized for more complex queries.

In the join operations of the $R-E-A$ categories in the REA data model, the focus also can be placed on the Resources (see Table II). In our example, the focus can only be on the Product table since it is the only resource in the simplified case. We can obtain Inventory Subsidiary information using the outer join operation on the Product table because we want to show all activities--receiving and/or shipping out, or inactivity--for each of the items in the Product table. However, to obtain Inventory Subsidiary, we must combine information from the database about goods receiving and shipping out. First, a natural join will be applied to tblProduct and tblGoodsShipping to obtain information from the database about goods shipped out (See Figure 1 as well). The natural join is used because the company can only ship whatever they have in the tblProduct. Since there is a Many-to-Many relationship between tblProduct and tblGoodsShipping, a relationship table is thus created. This relationship table will serve as the basis of the information for the result table in the natural join since it is the "Many" side of the relationships to both tblProduct and tblGoodsShipping. Second, an outer join operation will be applied to tblProduct and tblGoodsReceiving, based on tblProduct, in order to include all records, both participative and non-participative (i.e., received and non-received), from the tblProduct. It would be a left (or right) outer join if tblProduct is on the left (or right). Finally, a union operation will be used to combine the results of these two join operations. QBE, as well as SQL, can be used to accomplish these tasks. (The join processes with detailed descriptions, are shown in Figure 4). For example, using QBE, we can first create a Make-Table query for the natural join operation after we create a permanent table object (e.g., InventorySubsidiary) to store goods shipped-out-information in the Inventory Subsidiary, and then create another Append query for the outer join operation to combine both goods shipped-out- and receivedinformation. The Inventory Subsidiary is retrieved after the result table is sorted, based on the items in the tblProduct and the date.

The focus can also be placed on the Agents in the join operations of the $R$-E-A categories (see Table II). The origin of Agent-type information includes Salesperson, Customer, Carrier, Employee, and Vendor. Examples of reports about this type of information are performance evaluations-related. The join process in such joins is similar to the outer join mentioned above, but because of space limitations, we will not go through any examples.

The second major type of entity combination involves two sequential events, $E$ - $E$, with an emphasis on the previously occurred event. This combination requires an outer join on the previously occurred event in the query and produces a result table containing all records, both participative and non-participative, from the previouslyoccurred event table. The purpose of performing this outer join is to obtain non-participative records as well as the participative records that do not meet a specific condition (e.g., quantity received does not equal quantity ordered). As a result, the participative records that do satisfy the condition will be screened out in the query operation. The origin of the Event $\rightarrow$ Event-type information includes Sales Ordering $\rightarrow$ Goods Shipping, Sales Invoicing $\rightarrow$ Sales Collecting, Purchase Ordering $\rightarrow$ Goods Receiving, and Goods Receiving $\rightarrow$ Purchase Disbursing. Reports that result from the use of this type of information are unfilled sale orders, A/R subsidiary, unfilled purchase orders, and A/P subsidiary.

The processes of the join operations for A/R Subsidiary are similar to those for Inventory Subsidiary (i.e., using Union in Figure 4). However, Figure 5 illustrates an example of A/R aging report by invoice instead. In this example, an outer join is performed on two result tables: one for the Invoice Summary information (involving natural joins with tables such as tblCustomer, tblSalesInvoicing, tblSIProd, and tblProduct, shown in Figure 2), and another for the Collection Summary information (involving a natural join on tblSalesCollecting and tblCustomer) based on the Invoice Summary which contains information about all of the invoices issued. Information contained in 
both result tables will be aggregated (i.e., GROUP BY and SUM) based on the Invoice\# before the outer join operation. A query condition will be set on the inequality between InvTotal and CkAmt (i.e., a balance remains on the invoice) to screen out invoices that have been paid in full (See the note in Figure 5 for detailed descriptions of the join operations.) This process also can be used to obtain inventory on-hand information.

\section{Conclusions And Future Research}

There are many other types of entity combinations that can be applied to AIS using the REA data model, such as $E-A, R-E$, or $R-A$ (these may exist in some cases, but they are not covered in the case used here, nor are many other needed accounting documents and reports). A presentation of the join operations involved in a complete case is definitely of interest but is not within the scope of this paper. We hope that the taxonomy of join operations presented will provide AIS end-users and educators with the needed general knowledge and understanding of join operations and their accounting applications in relational database queries.

We have demonstrated the join applications applicable to the $R E A$ data model, but this approach can be applied to any $E-R$ data model using categories such as Objects, Events, Persons, Places, and Concepts (McFadden, Hoffer, and Prescott 1999). A similar taxonomy consisting of combinations of the categorical elements, focused entity, origin of the information, and documents and reports, can be created accordingly. As a matter of fact, the results in this case may be similar since the categories in the $R E A$ and the $E-R$ are interchangeable (e.g., $R e$ sources $=$ Objects, Events $=$ Events, and Agents $=$ Persons).

\section{Footnotes}

1 Referential integrity ensures that the foreign key (i.e., Zip Code) of the Customer table is a reference to the primary key (i.e., ZipID) of the Zip Code table.

2 Since a Many-to-Many relationship cannot be directly implemented in relational database management systems, a relationship/bridge table is created to bridge this relationship. As a result, most of the relationships (if not all) in relational database will be One-to-Many.

3 A Zip Code table consisting of City and State may also be involved in the joins if they are needed in the Sales Journal. To simplify the demonstration, however, this particular table is excluded. Some customer and product information is also excluded in the result table.

$4 \quad$ Foreign keys are included to establish relationships between two tables.

\section{References}

1. Bain, C. E., A. I. Blankley, and L. M. Smith. 2002. "An examination of topical coverage for the first accounting information systems course”. Journal of Information Systems (Fall): 143-164.

2. Borthick, A. F., P. L. Bowen, S. Liew, and F. H. Rohde. 2001. "The effects of normalization on end user query errors: an experimental evaluation". International Journal of Accounting Information Systems (December): 195-223.

3. Bowen, P. L., and F. H. Rohde. 2002. "Further evidence of the effects of normalization on end-user query errors: an experimental evaluation". International Journal of Accounting Information Systems (December): 255-290.

4. Chan, H. C., B. C. Wei, and K. K. Wei. 1999. "Three important determinants of user performance for database retrieval". International Journal of Human-Computer Study (November): 895-918.

5. Desai, B. C. 1990. An Introduction to Database Systems. St. Paul, MN: West Publishing Co.

6. Dunn, C. L., and W. E. McCarthy. 1997. "The REA accounting model: intellectual heritage and prospects for progress". Journal of Information Systems (Spring): 31-51.

7. El-Masri, R., and S. B. Navathe, 1989. Fundamentals of Database Systems. Menlo Park, CA: Benjamin/Cummings.

8. Hollander, A. S., E. L. Denna, and J. O. Cherrington. 2000. Accounting, Information Technology, and Business Solutions. 2nd ed., Burr Ridge, IL: Irwin/McGraw-Hill. 
9. Kambayashi, Y. 1985. "Processing cyclic queries". In Query Processing in Database Systems, edited by W. Kim, D. S. Reiner, and D. S. Batory, 62-78. New York, NY: Springer-Verlag.

10. McFadden, F. R., J. A. Hoffer, and M. B. Prescott. 1999. Modern Database Management. Reading, MA: Addison-Wesley.

11. Maier, D. 1983. The Theory of Relational Databases. Rockville, MD Computer Science Press.

12. McCarthy, W. E. 1979. "An entity-relationship view of accounting models". The Accounting Review (October): $667-686$.

13. __ 1982. "The REA accounting model: a generalized framework for accounting systems in a shared data environment". The Accounting Review (July): 554-578.

14. Mishra, P. and M. H. Eich. 1992. "Join processing in relational databases". ACM Computing Surveys (March): 63-113.

15. Morishita, S. 1997. “Avoiding cartesian products for multiple joins”. Journal of the ACM (January): 57-85.

16. O'Donnell E., and J. S. David. 2000. "How information systems influence user decisions: a research framework and literature review". International Journal of Accounting Information Systems (December): 178-203.

17. Olsen, D. H. 2000. "Accounting database design and SQL implementation revisited". The Review of Accounting Information Systems (Winter): 53-58.

18. Perrizo, W., J. Y. Lin, and W. Hoffman. 1989. "Algorithms for distributed query processing in broadcast local area networks". IEEE Transactions on Knowledge Data Engineering (June): 215-225.

19. Pillsbury, C. M., and T. J. Wang. 2003. "More on supplemental materials for database management system knowledge and skills in the accounting information system course". The Review of Business Information Systems (Winter): 11-22.

20. Ramakrishnan, R., and J. Gehrke. 2000. Database Management Systems. Boston, MA: McGraw Hill.

21. Wang, T. J., H. Du, and H. Lee. 2002. "A user-oriented approach to data modeling: a blueprint for generating financial statements and other accounting-related documents and reports". The Review of Business Information Systems (Fall): 77-92.

22. Weber, R. 1986. "Data models research in accounting: an evaluation of wholesale distribution software". The Accounting Review (July): 498-519.

23. Yoo, H., and S. Lafortune. 1989. "An intelligent search method for query optimization by semi-joins". IEEE Transactions on Knowledge Data Engineering (June): 226-237.

Table 1

Entity Types for Acquisition/Payment and Sales/Collection Processes of a Simple Merchandise Company

\begin{tabular}{|c|c|c|c|}
\hline \multirow{2}{*}{$\begin{array}{l}\text { Types of } \\
\text { Business } \\
\text { Processes }\end{array}$} & \multicolumn{3}{|c|}{ Entity Types Based on REA Data Model } \\
\hline & Resource $^{\#}$ & Event & Agent \\
\hline $\begin{array}{l}\text { Acquisition/ } \\
\text { Payment }\end{array}$ & Product (tblProduct) & $\begin{array}{l}\text { Purchase Ordering (tblPO) } \\
\text { Goods Receiving (tblGoodsReceiving) } \\
\text { Purchase Invoicing (tblPurchaseInvoice)* } \\
\text { Purchase Payment Disbursing (tblPayment) }\end{array}$ & $\begin{array}{l}\text { Vendor (tblVendor) } \\
\text { Employee (tblEmployee) }\end{array}$ \\
\hline Sales/Collection & Product (tblProduct) & $\begin{array}{l}\text { Sales Ordering (tblSO) } \\
\text { Goods Shipping (tblGoodsShipping) } \\
\text { Sales Invoicing (tblSalesInvoicing) } \\
\text { Sales Collecting (tblCashReceiving) }\end{array}$ & $\begin{array}{l}\text { Salesperson (tblSalesperson) } \\
\text { Customer (tblCustomer) } \\
\text { Carrier (tblCarrier) } \\
\text { Employee (tblEmployee) }\end{array}$ \\
\hline
\end{tabular}

* This is an optional entity since the information can be derived from tblPO and tblGoodsReceiving (i.e., the matched records).

\# To simplify the illustrations, suppose there is only one Cash account involved in the case. As a result, there is no need to create a Cash entity. The cash related information can be gathered from Purchase Payment Disbursing (outflow) and Sales Collecting (inflow) events. 
Figure 1

A Simplified REA Data Model For A Simple Merchandise Company

\section{ACQUISITION/PAYMENT PROCESSES}

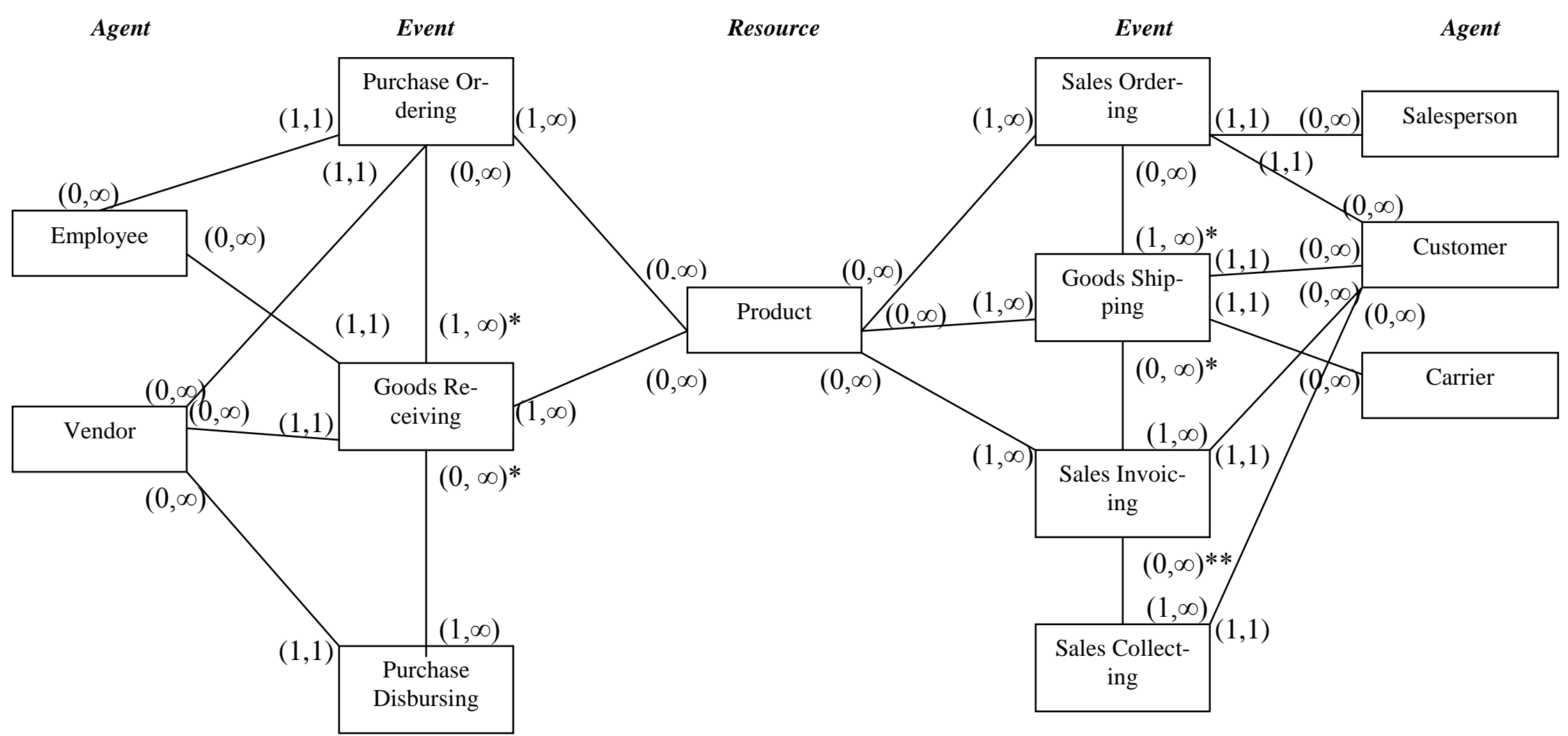

* The maximum cardinality may be 1 in some other cases.

** Customer may have a choice to pay partial payment on the invoice. 
Table 2

Taxonomy Of Join Operation In The REA Data Model

\begin{tabular}{|c|c|c|c|c|c|}
\hline $\begin{array}{c}\text { Entity Cat- } \\
\text { egories } \\
\text { Involved }\end{array}$ & Entity Focused & $\begin{array}{l}\text { Join Type } \\
\text { Based } \dagger\end{array}$ & Origin of the Information & Documents & Reports \\
\hline \multirow{13}{*}{$R-E-A$} & \multirow{7}{*}{$R-E^{*}-A$} & \multirow{7}{*}{ Natural Join } & Purchase Ordering & Purchase Order & Purchase Order Summary \\
\hline & & & Goods Receiving & Receiving Report & Receiving Summary \\
\hline & & & Purchase Disbursing & Check & Cash Disbursement Journal \\
\hline & & & Sales Ordering & Sales Order & Sales Order Summary \\
\hline & & & Goods Shipping & Bill of Lading & Shipping Summary \\
\hline & & & Sales Invoicing & Sales Invoice & Sales Journal \\
\hline & & & Sales Collecting & Cash Receipts & Cash Receipt Journal \\
\hline & $(A-E-) R^{*}-E-A$ & $\begin{array}{c}\text { Outer \& Natural } \\
\text { Joins }\end{array}$ & Product & Price List & Inventory Subsidiary \\
\hline & \multirow{5}{*}{ R-E-A* } & \multirow{5}{*}{$\begin{array}{c}\text { Outer \& Natural } \\
\text { Joins }\end{array}$} & Salesperson & & \multirow{5}{*}{ Performance Evaluation } \\
\hline & & & Customer & & \\
\hline & & & Carrier & & \\
\hline & & & Employee & & \\
\hline & & & Vendor & & \\
\hline \multirow{5}{*}{$E-E$} & \multirow{4}{*}{$\mathrm{E}_{1}^{*}-\mathrm{E}_{2}$} & \multirow{4}{*}{$\begin{array}{c}\text { Outer \& Natural } \\
\text { Joins }\end{array}$} & Sales Ordering \& Goods Shipping & & Unfilled Sales Orders \\
\hline & & & Sales Invoicing \& Collecting & & $\begin{array}{c}\text { A/R Subsidiary } \\
\text { A/R Aging Report }\end{array}$ \\
\hline & & & Purchase Ordering \& Goods Receiving & & Unfilled Purchase Orders \\
\hline & & & Goods Receiving \& Purchase Disbursing & & A/P Subsidiary \\
\hline & $\mathrm{E}_{1}-\mathrm{E}_{2}^{*}$ & $\begin{array}{c}\text { Outer \& Natural } \\
\text { Joins }\end{array}$ & Purchase Ordering \& Goods Receiving & & Purchase Journal \\
\hline
\end{tabular}

* Denotes that it is the focus of the join in the query.

$\dagger \quad$ Indicates the join operation based on the focused entity (although there may be other join operations involved in the query). 
Figure 2

Natural Join: A Sales Journal Example

tblProduct (Resource)

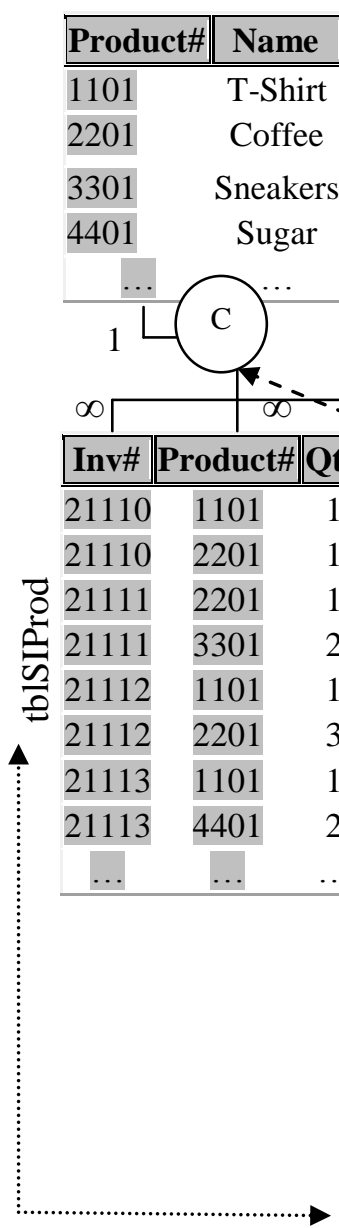

Note:

The Result Tabìe of thè Natural'Joins

\begin{tabular}{|c|c|c|c|c|c|c|c|c|}
\hline Inv\# & Product\# & Qty & Date & Name & B/L\# & Name & Price & $\ldots$ \\
\hline 21110 & 1101 & 1 & \multicolumn{3}{|c|}{ 3/10/2003m Nbucyustohes3 } & \multicolumn{2}{|c|}{ FidbinttblProd $\$ 6 t 00$} & $\ldots$ \\
\hline 21110 & 2201 & 1 & $3 / 10 / 2003$ & Mary & 10053 & Coffee & $\$ 2.00$ & $\ldots$ \\
\hline 21111 & 2201 & 1 & $3 / 18 / 2003$ & John & 21124 & Coffee & $\$ 2.00$ & $\ldots$ \\
\hline m21blsta & alesßrablici & & $3 / 18 / 2003$ & John & 21124 & Sneakers & $\$ 10.00$ & $\ldots$ \\
\hline 21112 & 1101 & 1 & $3 / 20 / 2003$ & John & 22146 & T-Shirt & $\$ 6.00$ & $\ldots$ \\
\hline 21112 & 2201 & 3 & $3 / 20 / 2003$ & John & 22146 & Coffee & $\$ 2.00$ & $\ldots$ \\
\hline 21113 & 1101 & 1 & $3 / 22 / 2003$ & Cherry & 24572 & T-Shirt & $\$ 6.00$ & $\ldots$ \\
\hline 21113 & 4401 & 2 & $3 / 22 / 2003$ & Cherry & 24572 & Sugar & $\$ 2.00$ & .. \\
\hline$\ldots$ & $\ldots$ & ... & ... & $\cdots$ & ... & $\cdots$ & $\cdots$ & .. \\
\hline
\end{tabular}

tblSalesInvoicing (Event)

\begin{tabular}{|c|c||c:|c|c|}
\hline Inv\# & Date & Customer\# & B/L\# & $\ldots$ \\
\hline \hline 21110 & $3 / 10 / 2003$ & 0001 & 10053 & $\ldots$ \\
21111 & $3 / 18 / 2003$ & 0002 & 21124 & $\ldots$ \\
21112 & $3 / 20 / 2003$ & 0002 & 22146 & $\ldots$ \\
21113 & $3 / 22 / 2003$ & 0004 & 24572 & $\ldots$ \\
$\ldots$ & $\ldots$ & $\ldots$ & $\ldots$ & $\ldots$ \\
\hline \multicolumn{5}{c}{$\infty$} \\
\end{tabular}


Figure 3

Natural Join: A Sales Journal Example Using QBE and SQL

A. Query-By-Example (QBE)

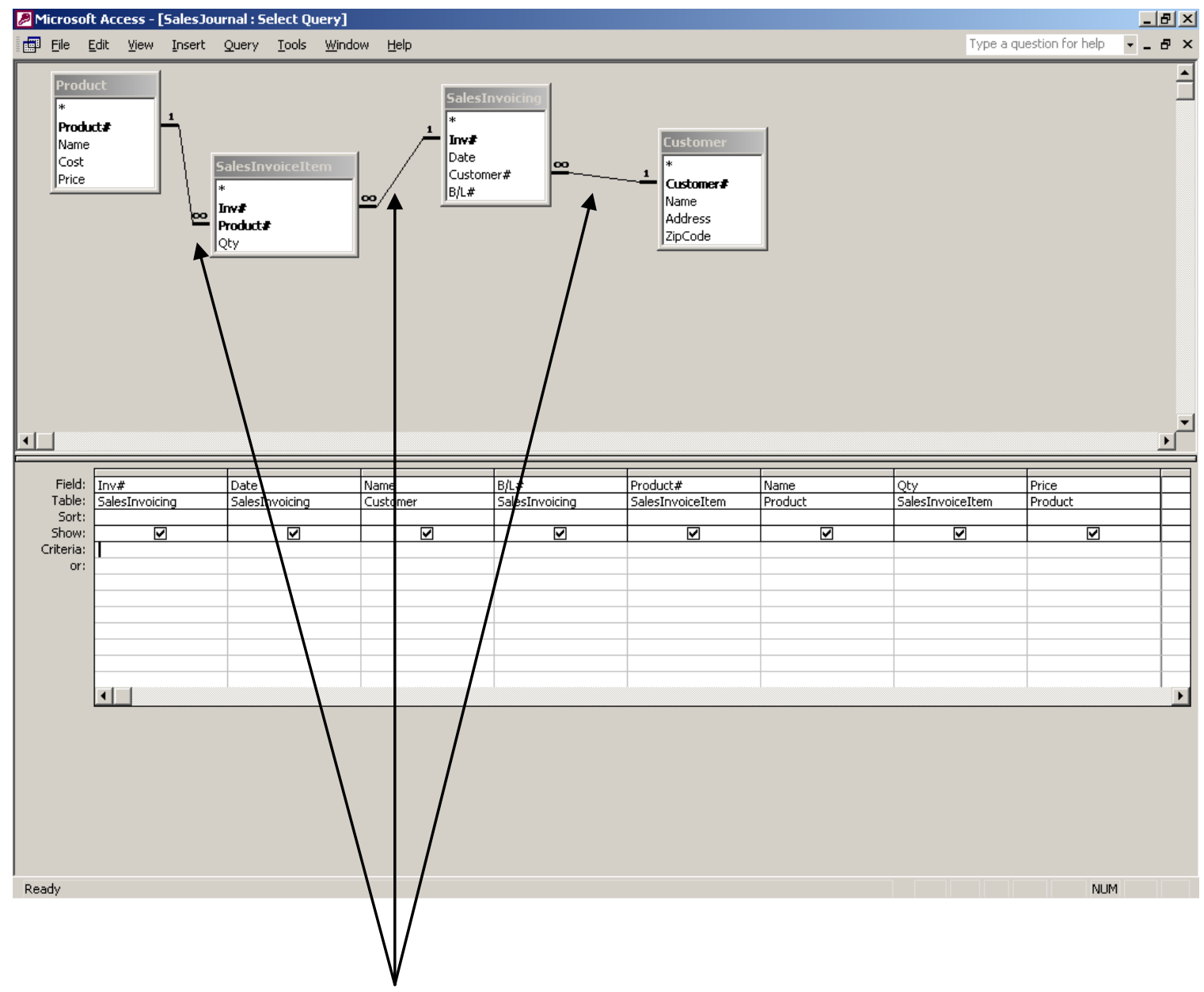

Applied the default join operation, natural join.

\section{B. Structured Query Language (SQL)}

SELECT SalesInvoicing.[Inv\#], SalesInvoicing.Date, Customer.Name,

SalesInvoicing.[B/L\#], SalesInvoiceItem.[Product\#], Product.Name,

SalesInvoiceItem.Qty, Product.Price

FROM (Customer INNER JOIN SalesInvoicing ON Customer.[Customer\#] =

SalesInvoicing.[Customer\#]) INNER JOIN (Product INNER JOIN

SalesInvoiceItem ON Product.[Product\#] = SalesInvoiceItem. $[$ Product\#])

ON SalesInvoicing.[Inv\#] = SalesInvoiceItem.[Inv\#]; 
Figure 4

Outer and Natural Joins: An Inventory Subsidiary Example

tblProduct (Resource)

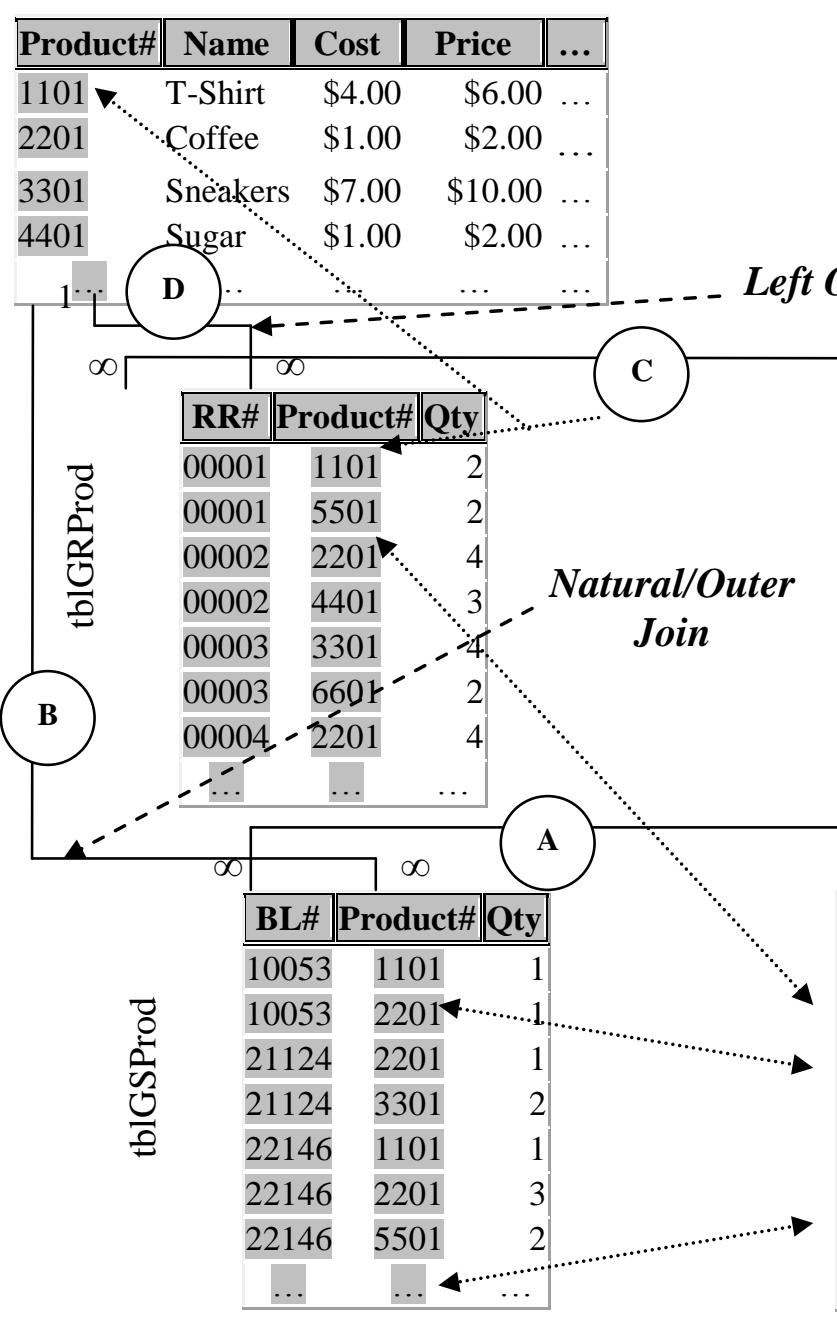

tblGoodsReceiving

\begin{tabular}{|c|c||c|}
\hline RR\# & Date & $\ldots$ \\
\hline \hline 00001 & $3 / 4 / 2003$ & $\ldots$ \\
00002 & $3 / 5 / 2003$ & $\ldots$ \\
00003 & $3 / 7 / 2003$ & $\ldots$ \\
00004 & $3 / 12 / 2003$ & $\ldots$ \\
$\ldots$ & $\ldots$ & $\ldots$ \\
\hline 1 & & \\
\hline
\end{tabular}

\section{tblGoodsShipping}

Natural Join

\begin{tabular}{|c|c|c|}
\hline BL\# & Date & $\ldots$ \\
\hline 10053 & $3 / 9 / 2003$ & $\cdots$ \\
\hline 21124 & $3 / 17 / 2003$ & \\
\hline 22146 & $3 / 19 / 2003$ & . \\
\hline$\ldots$ & $\ldots$ & $\ldots$ \\
\hline
\end{tabular}

Natural Join

1

The Result Table of the Union

\begin{tabular}{|c|c|c|c|}
\hline Product\# & Name & Date & QtyRecd QtyShip \\
\hline
\end{tabular}

\begin{tabular}{|l|l|l|l|}
\hline 1101 & T-Shirt & $3 / 4 / 2003$ & 2
\end{tabular}

\begin{tabular}{l|l|l}
1101 & T-Shirt $3 / 4 / 2003$
\end{tabular}

1101

T-Shirt

$3 / 9 / 2003$

1101

2201

T-Shirt

$3 / 19 / 2003$

Coffee

$3 / 5 / 2003$

2201

Coffee

$3 / 12 / 2003$

2201

2201

Coffee

$3 / 9 / 2003$

Coffee

$3 / 17 / 2003$

\begin{tabular}{|c|c|}
\hline 2 & 0 \\
\hline 0 & 1 \\
\hline 0 & 1 \\
\hline 4 & 0 \\
\hline 4 & 0 \\
\hline 0 & 1 \\
\hline 0 & 1 \\
\hline
\end{tabular}

Note:

A Union of two result tables from four join operations is required to obtain information of Inventory Subsidiary from five tables, such as tblGoodsReceiving, tbleProduct, tblGRProd, tblGoodsShipping, and tblGSProd. Both tblGRProd and tblGSProd are relationship tables.

A natural join, marked as $\mathbf{A}$, is performed on tblGoodsShipping and tblGSProd. The information of shipping date and others from tblGoodsShipping is merged with the information in tblGSProd based on the referenced values stored in BL\#. A natural join (or an outer join based on tblProduct), marked as $\mathbf{B}$, is performed on tblProduct and the result table from the previous join based on the referenced values stored in Product\#. The result table will contain information about goods shipping.

A natural join, marked as $\mathbf{C}$, is performed on tblGoodsReceiving and tblGRProd. The information of receiving date and others from tblGoodsReceiving is merged with the information in tblGRProd based on the referenced values stored in RR\#. An outer join based on tblProduct, marked as $\mathbf{D}$, is performed on tblProduct and the result table from the previous join based on the referenced values stored in Product\#. The result table will contain information of ALL goods receiving or not. Finally, a Union operation is performed to combine both result tables. 
Figure 5

Outer and Natural Joins: A/R Aging Report Example

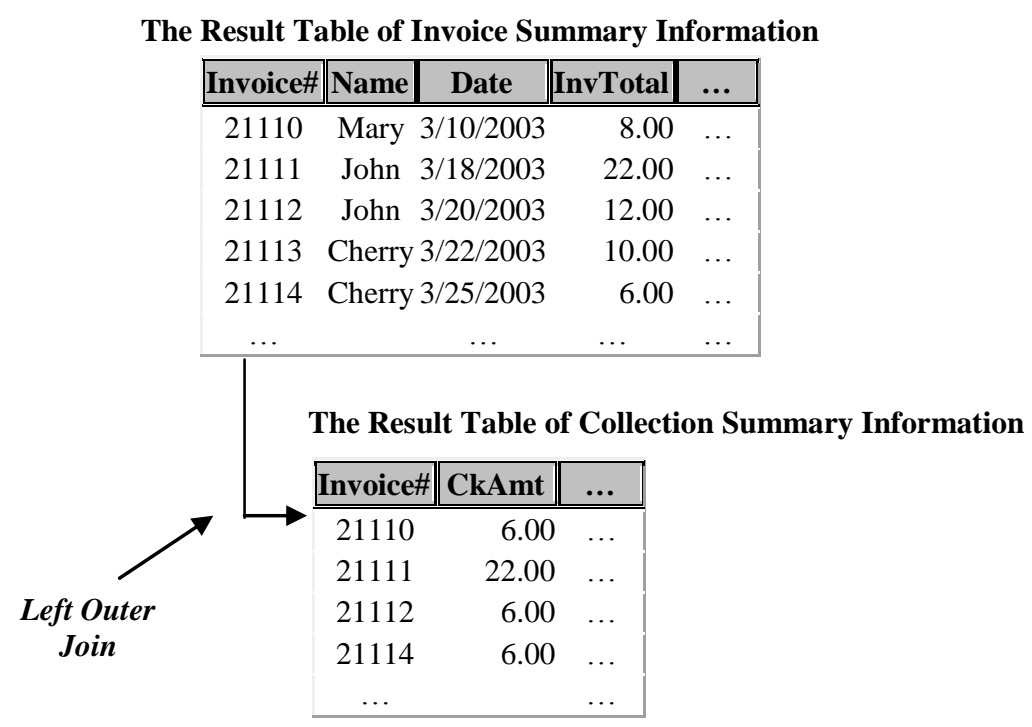

The Result Table of the Left Outer Join

\begin{tabular}{|c|c||c|r|r||c|}
\hline Invoice\# & Name & Date & InvTotal & CkAmt & $\ldots$ \\
\hline \hline 21110 & Mary & $3 / 10 / 2003$ & 8.00 & 6.00 & $\ldots$ \\
21111 & John & $3 / 18 / 2003$ & 22.00 & 22.00 & $\ldots$ \\
21112 & John & $3 / 20 / 2003$ & 12.00 & 6.00 & $\ldots$ \\
21113 & Cherry & $3 / 22 / 2003$ & 10.00 & & $\ldots$ \\
21114 & Cherry & $3 / 25 / 2003$ & 6.00 & 6.00 & $\ldots$ \\
$\ldots$ & $\ldots$ & $\ldots$ & $\ldots$ & $\ldots$ & $\ldots$ \\
\hline
\end{tabular}

Note:

The result table of Invoice Summary information was obtained using aggregate functions (GROUP BY and SUM) based on the Invoice\#, on the result of three natural joins on tblCustomer, tblSalesInvoicing, tblSIProd, and tblProduct (shown in Figure 2).

The result table of Collection Summary information was obtained using aggregate functions based on the Invoice\#, on the result of a natural join on tblCustomer and tblSalesCollecting.

The A/R aging report by invoice is generated using an outer join on the previous result tables with the Invoice Summary table serving as the basis for the join since it contains all invoices issued so far. All invoices in the Invoice Summary are included, whether it is participative or non-participative in the join with the Collection Summary table, in the final result table. 
Notes 